\title{
Manfred $\mathrm{H}$. Wiegandt »Ein Schatten über der Legitimität der Wahl«
}

\author{
Zur Kür des US-Präsidenten durch den \\ Supreme Court
}

Nicht weniger als 36 Tage vergingen, bis die Wähler, die bei den amerikanischen Präsidentschaftswahlen vom 7. November ihre Stimmzctel in die Wahlurnen geworfen hatcen, wußrcn, daß der Rcpublikaner George W. Bush ihr neleer Präsident sein würde. Ob die Mehrheir der Amerikaner oder auch nur die Mehrheit der an der Wahl teilnehmenden US-Bürger - nur erwa die Hälfte der Wahlberechtigten tut dies - ihn als ıhren neuen Präsidenten wollen, wird man mit ciniger Berechtigung bezweifeln dürfen. Nicht nur stimmten s $2 \%$ derjenigen, deren Stimmzetrel als gülcig gewerter wurden, für einen anderen Kandidaten, sondern auch die relative Mebrheit der gülugen Stimmen entíel auf den demokracischen Gegenkandidaten, Al Gore.' Der Sieg eines Kandidaten für das Amt des bedeutendsten Staars- und Regierungsoberhauptes der Welt ist jedoch nicht das Ergebnis eines streng demokratischen, nach dem Grundsacz wonc man, one vote* vor sich gehenden Wahlaktes, sondern folgi den archaischen Regeln einer über zweihunders Jahre alıen, für cine Union von damals dreizehn unabhängig gewordenen Staaten mit ciner relativ geringen Bevölkerungszahi konzipierten Verfassung. Diese Verfassung sah und sieht selbst heute noch nicht einmal die Volkswahl des Präsidenten vor, sondern läßt das Staztsoberhaupt in einem indirekten Wahlverfahren durch von den Einzelstaaten bestimmte Elektoren küren. Nach Artikel II $\$ 1$ der insoweir niemals geänderten US-Verfassung von 1787 ernennt jeder Einzelstaac nin einer durch seinen Gesetzgeber bestimmten Weise Elektoren, deren Anzahl sich nach der Summe der diesem Staat zustehenden Kongreßabgeordneten bestimmt. ${ }^{2}$ Alle mittenvejle fünfzig US-Bundesstaaten und auch der keinem Staate zugchörige District of Columbia, der die Hauptstadt Washington umfaßr und dem nach der Verlassung zwar keine Kongreßabgeordneten, aber Elektoren zustehen, haben sich inzwischen für die unmitcelbare Volkswahl der sie repräsenticrenden Elektoren entschicden. Wic diese Wahl im einzelnen abläufr, das war - zumindest bisher - nahezu gänzlich einc Angelegenheic für die einzelstaatliche Gesetzgebung. Fast uniform haben sich die Gliedstaaten jeweils entschieden, daß der Kandidat, der die relative Mebrheit bei dieser Volkswahl bekommt, auch alle Elektorcnstimmen des Staaces erhält ( The winner cakes it all.«). Dieses System macht es möglich, daß ein Kandidat, der nicht die Mehrheit der Seimmen insgesamt erhält, dennoch dic absolutc Mehrheis im Wahlmännergremium, dem Electoral College, crringt. Dies war zwar seir übcr hundert Jahren nicht mehr vorgckommen, ist aber in einem System wie dem amerikanischen, bei dem die bevölkerungsarmen Staaten auch bei der Präsident-

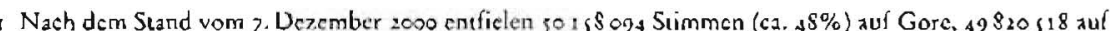
Bush (ca. $8 \%$ ), 2703722 Stimmen (ca. $3 \%$ ) auf Ralph Nader von der Grinen Parei und $43 \$ 407$ (unter 1\%) zuf Patrick Buchanan, der fur dic von Ross Perot einstmals ins Leben gerufene Reformpartc; kandidierte (Quelle: C-Span).

2 Die relevante Passage in Art. Il $\$ 2$ US-Verfassung lautct wörtich: "Each State shall appoint, in such Manner as the Legislature thereof may direct, a Number of Electors, equal to the whole Number of Senators and Representatives to which the State may be entitled in the Congress ...* 
schaftswahl überrepräsentiert sind, durchaus angclegr. ${ }^{3}$ Zwar wird man dem Umstand, daßß eis Kandidat nicht die wenigstens relative Mchrheit der Stimmen, die Mehrheit des spopular vorex, erhielt, keine besondere Legitimationsminderung scines Mandats entnelimen können, solange sich dic Diskrepanz zur Stimmenzahl des Verlierers vergleichsweise gering ausnimmt (hier waren es ca. 337000 Stimmen und damir weir unter $1 \%$ ), denn schlicßlich wird der Wahlkampf strategisch niche mit dem prinzipicllen Ziel geführh, die Mehrheit der Wählerstimmen zu gewinnen, sondern mit dem Ziel, die Mehrheit der Elektoren auf sich zu vereinigen. Von daher wird man nich genau sagen können, wie sich ein mit einem anderen Ziel gefülurter Wahlkampf auf das Stimmenergebnis ausgewirkt hätte."

Die Tarsache, daß der Kandidar mir der - wenigstens in absoluten Zahlen - klar geringeren Anzahl von Wählern hinter sich dennoch gewinnt, wird aber in demokrarisch-legirimacorischer Hinsicht dann zweifelhafe, wenn der Sieg an einigen hundert Stimmen in cinem cinzelnen Stzat hängt, der mit seinen 25 Elektoren das Blatt wender (Gore fehlten ohne Florida nur vier Stimmen zur Mehrheit im Electoral College). Wenn schon Zünglein an der Waage, so wärc es doch wünschenswert gewesen, wenn dicse relacive Mehrheic wenigstens dem lecztlich Erfolgreichen eindeutig zuzuordnen gewcsen wäre. Gerade dies war aber mitnichten der Fall. Erstens war die Mehrheit bei insgesame knapp 6 Millionen gültigen Stimmen in Florida nur eine solche von wenigen hunder Stimmen. Zweirens gab es eine Reihe von Vorfâllen, die dem Verlierer recht eindcutigerweise ihm zugedachie Scimmen vorenthielten. Nocorischwar dabei der sog. "Butcerfly Ballor $\alpha$ (Schmetterlingsstimmzetrel)' in Palm Beach County, einer Hochburg der Demokraten. Der Stimmzetrel war dort - mic Billigung einer demokrarischen Wahlausschußvorsirzenden - so ausgelegr, daß Wähler, die Al Gore ihre Stimme geben wollten, leichr aus Versehen dem Rechtsaußen Buchanan ihre Stimme gaben, was zur Folge hatce, daß dicser vielfach als Antisemit angesehene Kandidac gerade in einem srark jüdisch-demokratisch geprägren Bezirk mehr als dreimal so viel Stimmen erhielt wie in jedem anderen Wahlbezirk in Florida. Es war offenkundig - und der Begünstigte selbsr gestand dies ein -, daß hier vielfach Leure versehentlich ihre Srimme Buchanan gegeben haten, obwolyl ihnen womöglich nichts ferner lag als dies. Ohne größere staristische Kompecenz zu besiczen, war leich zu folgern, daß ohne diesen Fehler Gore mehr als 2000 weitere Srimmen zugefallen wären, die das Ergebnis bereirs zu seinen Gunsten verändert härten. Darüber hinaus war die Anzaht der ungültigen Doppelstimmen hier weic höher als irgendwo sonst in Florida, was es als sehr nahelicgend erscheinen läßr, daß vielc Wähler ihren Irrtum

3 Dic in Art.II $\$ 2$ US-Verfassung beschriebene Zusammensetzung des Electoral College fuhrt dazu, daß kleine Staaecn, denen nach ihrem Bevölkerungsanteil nur cin Mirglied des Repräsentantenhauses zustcht, drei Wahlmänner zukommen, denn jedem Staat stehen genau zwei Senatoren zu. Das Repräsentantenhaus besteht seit cinem 1929 verabschiedeten Gesetz ("Permanene Apportionment Act *) aus 435 Abgeordneten, und nach der Verfassung steht jedem Staat mindestens ein Abgeordneter zu $(A r .1 \$ 2)$. Dinit ergibt sich im Electoral College eine leichte proportionale Ungleichheit zu Lasten der bevölkerungsrcichen Staaten, auch wenn diese bei weitem nicht so groß ist wie im US-Senat. Siche ausfülrlicher dazu Marired H. Wiegandt, Das denokratische Defizit der USA, Recht und Politik 1997, S. 162-169.

4 Mutmaßen läßt sich nur, daß ein Kandidat, der weitere, bei dieser Wahl nicht walilende Massen in bevölkerungsrcichen Staaten wie Kalifornien und Texas, dic unumstritten waren, häre mobilisieren können, im Vorecil gervesen wărc.

s Die Kandidaten waren auf diesem Stimmzettel in zwei Spalten untercinander aufgelistet. Dic erste Kolumne begann mit Bush, an zweiter Stelle folgte Gore und dann weitere Kandidazen. Anstatt alle Kandidaten untereinander aufzulisten, wurde dann aber eine zweite Spalıe begonnen, in der Buchanan als erster aufgeführt war. Der Wähler mußte die Stinme für den von ihm gewünschten Kandidaten dadurch abgeben, daß erin der Mitue (zwischen den beiden Spalten) das diesem Kandidaen durch einen kleinen Pfeil zugewiesene Loch mit einem dafür vorgeschenen Stift einstanzte. Während das erste Loch für Bush bestimmt war, gehörne das zweice nicht zu Gore, sondern zu dem in der zuecten Spalte als erster aufgeführeen Buchanan. Eine Klage von (demokratischen) Wählern zuf Feststellut:g, daf dieser Stimmzettel gegen das Wahlgesetz Floridas versticß, nach dem Stimmzeud aklar und unzwcideurig a scin mússen. scheitere vor den Gerichten Floridas. 
erkannten und eine zwcite Stimme für Gore abgaben, dadurch ihren Stimmzetrcl aber ungültig werden beßen. Hatten sie sicl korrekt verhalten und einen neuen Stimmzectel verlangt, wäre der Gore-Vorsprung wohl um cinige weitere Tausend Srimmen gewachsen. Sicherlich, formalrechtlich wird man sich auf den Standpunkt stellen können, daß diese Wähler ibre Fehler hätten vermeiden oder korrigieren können und damic selbst für die Konsequenzen ihres unaufmerksaınen Verhaltens verantwortlich sind. Das macht die Tarsache, daß der legicime Gewinner im Lande nur auf Grund rechnischer Mängel im Wahlverfahren in einem Staat "zufällig « nicht gewonnen hat, nicht weniger makaber. Hinzu kommen Berichte, daß in vielen Orsen in Florida schwarze (und in ihrer Mehrzahl demokratische) Wahler in unterschiedlicher Weise an der Stimmabgabe gehindert worden sein sollen." In zwei anderen Bezirken mit republikanischer Verwaltung hatten die Wablleiter/-innen republikanischen Parteiarbeitern erlaubs, unzulänglich ausgefüllıe Briefwahlanträge nachzubessern, indem sie die korrekte Wählerregistricrungsnummer im Computer nachgucken und in den Bricfwahlantrag nachtragen durften. ${ }^{7}$ Die Republikaner beschwerten sich andererseits darüber, daß die anerikanischen Fernsehanstalten aufgrund von Befragungen von Wählern nach Verlassen der Wabllokale Al Gore zu frülı (und fälschlicherrveise) als Sieger in Florida proklamiert hatten, weswcgen eine Reihe potentieller BushWähler zehn Minuren vor Schließung der Wahllokale in dem Bereich Floridas, der einer anderen Zeitzone angehörte und in dem die Wahllokale deshalb noch geöffnet waren, nicht rehr zur Walıl gegangen seien. ${ }^{8}$

Während es recht offensichelich schien, daß die genannten Probleme Gore den nimmt man den Wählerwillen als Maßstab - legicimen Wahlsieg gekostec hatten, richecte sich das wochenlange Tauziehen im Wesentlichen auf die Art und Weise des Nachzählens bzw. Zählens der abgegebenen Srimmen. Bemerkr sei vorøeg, daß die Srimmzertel in den 67 Bezirken des Staates Florida unterschiedlich gestalter waren und darüber hinaus die Stimmabgabe sowie das Zählen und Nachzählen der Srimmen in recht unterschiedlicher Weise vonstatten ging. In den besonders umstrittenen demokracischen Hochburgen Miami-Dade, Broward, Palm Beach und Volusia County wurden Stanzkarten verwender, auf denen die Wähler neben ihrem Kandidacen mit einem Stift kleine vorgestanzte Chips (sog. »chads $\alpha$ ) ausstechen mußten. Die Lochkarten wurden dann von recht anciken Wahlmaschinen auromatisch gezählt. Auf der anderen Seite gab es Bezirke - meist reichere, in denen man das moderne Wahlgerär anzuschaffen in der Lage war -, in denen Felder neben dem gexünschten Kandidaten mit einem Bleistifr markier werden mußcen. Diese dann von cinem oprischen Lesegerät gelesenen Stimmzettel wiesen eine wesentlich geringere Anfälligkeir für Wählerirrtum beim Markieren der Srimmen auf, weswegen die Anzahl der ungültigen Stimmen in diesen Bezirken um ein Vielfaches geringer war. Das Wahlrecht des Staates Florida sah für den Fall eincr Stimmendifferenz zwischen dem siegreichen und dem uncerlegenen Kandidacen von weniger als $0,5 \%$ ein automarisches Nachzählen der Stimmen vor. Dieses Nachzählen wurde in den meisten Wahlbezirken maschinell, in einigen aber auch manuell vorgenommen. Nach diesem ersten gesetzlich vorgeschriebenen Nachzählen reduzierte sich der Stimmenvorsprung George W. Bushs vor Al Gore von $17 \$_{4}$ auf uncer 1000 Stimmen.

6 Ironischerweise wird es nun die Bush-Regierung sein, dic diese Vorwürfe untersuchen wird.

7 Eine Vorschrift, dic Briefwähler dazu zwingt, in ihrem Antrag auf Bricfwahl ihre Wählerregistrierungsnummer anzugeben, war crst kürzlich in das Wahlgesetz aufgenommen worden, um den in der Vergangenheit bei der Briefwahl festgestellten zahlreichen Manipulationen entgegenzuwirken.

8 Daß dieser Fehler von Bushs Wahlkampfteam als absichuliche Einflußnahme der angeblich liberalen Medien zugunsten Gores bezeichnet wurde, ist aber angesichts des Irrtums aller Beteiligter, auch der konservativen Anstalten, an den Haaren herbeigezogen. Fraglich ist überdies, ob dieser Umstand nur potenticlle Bush-Wähler abgehalten hat, noch in den Iexten Minuten zu den Urnen zu gehen. 
Nun begann das eigentliche Drama. Das Gorc-Tcam nutzte die Vorschriften des Wahlrechts von Florida und beantragce das manuelle Nachzählen der Stimmen in Miami-Dadc, Palm Beach, Volusia und Broward County, eingestandenermaßen demokratischen Hochburgen, in denen es erwartet werden konnte, daß das manuelle Überprüfen von bistveilen von den Maschinen niche lesbaren, nur teilweise ausgestanzzen oder gar nur eingedrückten oder ausgebeulren, aber nicht durchgestochenen "chads" relativ gesehen erheblich mehr Gore- als Bush-Zugewinne ergeben würde. Dies war auch der Punkt, an dem die uncerschiedlichen Strategien der beiden politischen Lager, die bis zum Schluß durchgehalten wurden, einserzren. Während das Gorc-Team propagiere, daß jede Stimme, die identifizierbar sei, auch gezälylt werden müsse, stellte sich das Bush-Team auf den Standpunkt, daß die Srimmen zweimal ausgezählı seien, und zwar von Maschinen, die keine subjekciven Präferenzen härten, und daß dieses Ergebnis - ähnlich dem Pfiff eines Schiedsrichrers - zu respektieren sei. Katherinc Harris, Secretary of State des Staates Florida und CoVorsizzende des Bush-Wahlkampfteams in Florida, deren Aufgabe es war, das amtliche Wahlergebnis in Florida festzuscellen, bemühte sich zunächsh das manuelle Nachzählen aufzuhalten und stellte sich sodann auf den Standpunkt, daß eine im Wahlgesetz vorgesehene Frist zur Feststellung des amelichen Wahlergebnisses absolut bindend sei, weswegen die geserzlich zulässigen manuellen Nachzählungen der Stimmzettel nicht mehr zu berücksichtigen seien, wenn sie bis zu diesem Tage nicht abgeschlossen seien. Die Vorschrift über den Termin der amelichen Bekannrgabe des Wahlergebnisses als absolure Ausschlußfrist zu behandeln, vertrug sich indes niche mit der Tatsache, daß die Frist für das Eingehen von Briefwahlstimmen später lag. ${ }^{9}$

Die Demokraten befürchteten, daß die erhofften Zugewinne bei der Handnachzăhlung durch die drohende Festscellung des offiziellen Wahlergebnisses durch Harris vergeblich scin würden, und klagten vor den Gerichten Floridas gegen Harris mir dem Zicl, die Frist für die Nachzählung auszuweiten. Gleichzeitig beschwerten sich die Bush-Leute konstant darüber, daß cs zuriefst unfair sei, daß Gore dic Handauszahhlung selektiv in demokratischen Hochburgen verlangt habe und so überproportionale Gore-Gewinne vorprogrammiert seien. Des weiteren rügten sic, daß von Wahlausschuß zu Wahlausschuß unterschiedliche und zum Teil wechselnde Standards bei der Auswertung der Stimmzectel angewandt würden, und dies unter der Oberaufsicht von zuncist mit Demokraten besetzten Wahvorständen. Der Oberste Gerichtshof von Florida, dessen sieben Mitglieder allesamt von demokratischen Vorgängern des derzeirigen republikanischen Gouverneurs und Bruders des Präsidentschaftskandidaten, Jeb Bush, ernannt worden sind, entschied diesen Rechrsstreir letzrlich dahin, daß die Frist zur Einreichung von Wahlcrgebnissen in den drei Nachzählbezirken von Harris zu verlängern sei. Das Gericht hatte auf diese Weise cinen Konflikt zwischen cinem Gesetz, das eine feste Frist für die Feststellung des amtlichen Endergebnisses vorsah, und einem späreren Gesecz, das dic Möglichkeit eines Walalprotestes mic folgender Handnachzählung ermöglichte, Jösen wollen. Von republikanischer Seice wurde dem Gericht jedoch nunmehr vorgehalten, daß es durch die Erweiterung der Frist in die Prärogative des Geseczgebers eingegriffen und eine unzulässige Rechtsfortbildung vorgenommen habe, was angesichrs Art. II $\$ 2$ der USviorfassung, wonach der Gesetzgeber des Einzelstaates das Verfahren für die Bestim-

9. Die Briefwăhler begünstigten wie envartet Gcorge Bush. Hier gab cs das l'roblem, daß Brichruahlstimmen, die von Miliärangehörigen in Ubersee abgegeben wurden, of nichı cinmal das Datum crkinnen licßen, weil sic über ein Militärpostfach cingingen. Die Tatsache, da $\{$ cinige Demokratcn und wols] auch solche aus dem Umield des Gore'schen Wahlkampfteams sich bemühten, Bricfwahlstimmen aus formellen Grunden für ungültig erklären zu lassen, gab dem Bush-Team sine Propagandawaffe in die Hand, da cs als unverscihlich angesehen wurde, den dem Vaterland Dienenden das Stimmrecht zu entzieher. 
mung der Elekroren regele, auch bundesverfassungsrechulich unzulässig sei. Bush legte vor dem U.S. Supreme Court Revision gegen dieses Urteil wegen der Verletzung des Gewahenteilungsprinzips, des Gleichheirssarzes und des Arc. II $\$ 2$ der USVerfassung cin.

Unterdessen begann ein sich über den Thanksgiving-Feiertag erstreckender Nachzähl-Mararhon, um dic vom Gerichr ncu bestımmre, aber immer noch extrem knapp bemessene Frisı einhalıen zu können. Broward Counry war der einzige Bezirk, der seine Stimmen mil nichr uncrheblichen Gore-Zugewinnen rechtzeitig der Secretary of State einreichte. Palm Beach schaffte es nicht, alke Stimmen nachzuzählen, und reichte ein teilweise korrigiertes Stimmenergebnis ein. Miami-Dade schließlich, das erst die von den Demokraten beantragte Handnaclizählung abgelehnt, dann aber doch begonnen harte, brach sie wieder ab mu dem Argument, daß sie niche in der Kürze der Zeit zu bervältigen sei, ${ }^{10}$ und bezog auch nicht dic bei der bis dahin durchgeführten Nachzählung sich ergebenden Scimmenveränderungen zugunsten Gores in das an Harris bcrichtete Endergebnis ein. Secrecary Harris korrigierce das ursprüngliche Ergebnis insoweit zugunsten Gores, als sie das Ergebnis der abgeschlossenen Nachzählung in Broward County bericksichrigre, ignorierte aber die Teilergebnisse aus Palm Beach und die (nicht offizicll gerneldecen) Teilergebnisse aus Miami-Dade.

Der U.S. Supreme Courc, dessen konservative Mehrheit sich in vergangenen Entscheidungen stets als Bewahrerin der Rechte der Einzelstaaten gegenüber Einflußnahme durch den Bundesstaat gcriert harte" und insoweit den einzelstaatlichen Gerichten in der Regel einen weiten Ermessensspielraum gewährc hatte, sich außerdem als nichtaktivistisches Gcricht herausgehalten hatte, das sich scheut, in politische Streitigkeiten einzugrcifen, nahm die Revision gegen die Entscheidung des Florida Supreme Courr für die meiscen Fachleute völlig überraschend zur Entscheidung an, beschränkte sich aber auf den bundesverfassungsrechclichen Aspekr des Art. Il $\$ 2$. Einc solche Eilannahme bedurfte einer Mehrheil des neunköpfigen Gerichts, und bereits hier wurde spekulier, daß eine konservarive Mehrheic im Supreme Courr aus policischen Gründen in den Prozeß eingreife. Dic Entscheidung selbst stelle dann aber einen einstimmig getragenen Kompromiß dar. Das Gericht verwies die Entscheidung an den Obersten Gerichtshof von Florida zurück und bat (lediglich) um Aufklärung, ob dessen Entscheidung eine juristische Lösung eines Konfliktes zwischen zwei widersprüchlichen Rechtsvorschriften gewesen sei oder ob das Gericht scine Entscheidung (etwa) auf die Verfassung des Staates Florida gcstützc habe, als es unter Hinweis auf diese Verfassung von cinem elementaren Recht der Bürger des Staates sprach, ihr Wahlrecht auszuüben. Außerdem sei nicht klar, inwieweit das Oberste Gericht von Florida bei seiner Entscheidung ein Bundesgesetz berücksichrigt habe, wonach die Bestimmung von Elektoren durch einen Staat für den U.S.Kongreß (der das Wahlergebnis im Electoral College fesizustellen hat) bindend ist,

1c Der Vorwurf, daß cs organisierter, cinschüchtetnder Protcst seitens republikanischer Demonstranten war, der den Wahlausschuß zur Einstellung der manucllen Nachzählung bewog, scheint indes die durchaus massiven Proteste republikanischer Aktivisten überzubewerten.

1. Die konservative $5: 4$-Mehrheit hat in vergangenen Jahreo kontinuterlich den Finfluß des Bundesstaates zunickgeschraubs, indem es Arr. I $\$ 8$ par. 3 der US-Veriassung, die sog. „Commerec Clauses, auf die scil der New Deal-Ära weiereichende bundesstaaliche Geserygebungskomperenzen gestürzt zu werden oflegten, dic vom Suprenc Court nichr hinterfragt wurden, immer enger ausgeleg ha. Dadurch wurde der Wirkungsbereich cuner Reihcs von Gesetzen, etwa im Umweitrecht, aber auch der Violenec Against Women Act, der Frauen eine bundesgesetzliche Handliabe gegen Vergewaltiger gab, eingeschrankt. Das Gericht hat unter dem Vorsitz von William Rehnquist, der 1983 von Reagan zum Chicf Justice gemache wurde, den Einzelstaaten außerdem weitreichende Immunität gegen Klagen zugesprochen, die auf bundesrecheliche Anspruchsgrundlagen gestütz, sind, so etwin im Bereich des Urheberrechis oder der Bürgerrechte. 
wenn diese Wahlmänner sechs Tage vor dem Zusammentreten des Electoral College von dem Einzelscaat bestimm sind (sog. "safe harbor"-Bestimmung). " Im Klarrexr hieß dies folgendes: Der Supreme Court würde die Neubestimmung der Frist für die Fesrstellung des amrlichen Endergebnisses nich als Überschreitung der richrerlichen Kompetenz ansehen, wenn das Gericht lediglich den Willen des Gesetzgebers in Florida im Hinblick auf die widerspnichlichen Geseczesvorschrifcen gedeuter hätte. Wenn das Gericht in Florida dem Geserzgeber aber Grenzen serze, erwa durch den Grundsacz des Vorrangs des Wählerwillens, so sei dies im Hinblick auf die USVerfassung nöglicherweise bedenklich, habe diese doch eine Komperenz an den einzelstaatlichen Gesetzgeber olıne jegliche Bindung übertragen wollen. Diese bereirs aus der ersten Entscheidung des U.S. Supreme Coun zur Wahlauszählung in Florida durchscheinende Auffassung ist juristisch problematisch, weil sie quasi einen einzelstaarlichen Geseczgeber aus dem Nichts fingiert. In Wirklichkeic isc cin Geserzgeber aber immer schon ein durch die Verfassung und die Rechtsvorschriften des betreffenden Einzelstaates konstruiertes Gebilde, das dem Recht - und nicht nur dem Verfassungsrecht - des betreffenden Staaces unterliegt. Stellt man sich aber diesen bindungslosen Geserzgeber des Sraates Florida vor, soll es wirklich so sein, daB der Oberste Gerichtshof selbstversiändliche demokrarische Prinzipien wie die Beachtung des Wählenwillens nicht in seine Überlegungen einbezichen können sollte? Hier offenbart sich cine sehr formaljuristische Sichrweise einiger oberster Bundesrichter. Die Verfassung wird stacisch beurteilc. Alle Enrwicklungen außerhalb der Verfassung innerhalb von über zweihundert Jahren dürfen bei der Verfassungsinterpretation keine Beachrung finden, wern sie niche durch eine ausdrückliche Vcrfassungsänderung direkten Eingang in den Text gefunden haben. Dies wäre ein nobler Grundsatz, wenn dadurch nicht dic Verfassung als konstitutioneller Rahmen einer real existiercnden Gesellschaft ad absurdum geführt würde. Die vor allem von Richter Antonin Scalia vertretene Verfassungsinterpretation, die nur aut den Willen des originären Verfassunggebers zu schauen vorgibn verschließt sich damit niche nur einer Fortentwicklung der Grundrechte, ctwa des Rechrs auf Privacheit, sondern auch einer demokracischen Anpassung des Verfassungscexces. Die Federalist Papers als murmaßlicher Ausdruck des Willens der Verfassunggeber im I 8. Jahrhundert enıfalten so weic stärkere juristische Wirkkrafe als die sich unter der Verfassung encwikkelnde moderne demokratische Praxis nach Ablauf von mehr als zwei Jahrhunderten. Die Verfassung wird so eher zum Korsett als zu einer Basis für demokratische Selbstregulierung eines Staatswesens.

Der Oberste Gerichtshof von Florida hatte die knappe Bemessung der neuen Frist für die amtliche Feststellung des Wahlergebnisses auch damit begrindet, daß das Recht in Florida zwei separate Phasen für die Infragestellung der Wahlergebnisse vorsah. Dic erste, mit der Handnachzählung verbundene, war der Wahlprotest. Danach müsse aber auch noch Zeit für die gerichtliche Wahlprüfung ("election contess «) bleiben, die darauf gerichter sei, das amrliche Wahlergebnis als unrichrig anzugreifen. Diese zentral am Tarsachengericht im Bezirk der Landeshauptstadt Tallahassee, Leon County, vorzunchmende Wahlanfechung war daher der nächste juristische Schrit des Gore-Teams. Die Anfechrung der Wahl aurde auf den im erst jüngst reformierten Wahlgeserz genannten Grund gestürze, daß eine Reile von ungültigen Stimmen in das Wahlergebnis aufgenommen worden seien, und (vor allem), daß eine Anzahl von gülrigen Stimmen nicht gewerter worden seien, dic das Wahlergebnis insgesame ändern oder zumindest in Zweifel ziehen würden. Als Grund führte das GoreTeam die als gülcige Srimmen identifizierten Srimmzetrel in Miami-Dade und Palm

12 3. US.C. S (1994). 
Beach County an, die von der Secretary of State nicht berücksichtigt worden seien, und cine Anzahl von ca. 9000 Stimmen in Miami-Dade, die von den Maschinen als "ohne Stimmabgabe bei der Präsidentschaftswahl « registrier worden waren und daher als ungülrig galcen (sog. wundervotes «), die aber mit großer Wahrscheinlichkeit bei einer Handauszählung in einer Vielzahl von Fällen einen Wählerwillen erkennen ließen, wie die anderen Handnachzählungen erwiesen hätten. Die Beweisaufnahme vor Richter Sauls's in Leon County konzentricrte sich vor allem auf rechnische Fragen, wie etwa auf die, welche Art von Stimmen bei welchem Wahlverhalten zu Markierungen des Stimmzectels führten, die von den Wahlmaschinen nicht als Suimme idenrifiziert würden, obwohl der Wähler habe wählen wollen. Naclı einer in langen Stunden an Wochenende durchgeführten Verhandlung entschied Sauls in allen Punkten gegen das Gore-Team und verweigerre vor allem, das angeborcne Beweismaterial, nämlich die aus den entsprechenden Counties herbeigeschafften Stimmzettel, in Augenschein zu nehmen. Gore legte erneur Revision ein, dic wiederum vom Obersten Gerichtshof von Florida angenommen wurde. Die republikanische Propaganda hatte bereits nach dem ersten Urtcil dieses Geriches die dortigen Richier als akrivistische Demokraten gebrandmarki und ihrem Urteil den Anschein der Parteilichkeir unterzulegen versuche, wie auch eines der Hauprargumence der Bush-Propaganda gegen eine Handnachzählung ${ }^{14}$ war, daß die Wahlvorstände in den betreffenden Bezirken hauptsächlich von Demokraren besetzr seien, von denen keine objektive Beurteilung erwarter werden könne. Die Demokraten führten an, daß Karherine Harris, die sich als Mirvorsitzende von Bushs Wahlkampikomicee hervorgeran hattc, sich eigentlich für befangen hätre erklären müssen. Eine unparteiische Entscheidung sei von ihr nicht zu erwarten.

Bevor der Obersce Gerichrshof über Gores Fall enrschied, wiesen zwei Tarsachengerichre Klagen von demokratischen Akrivisten's gegen dic Handhabung der Briefwahlanträge republikanischer Wähler in Martin und Seminolc County zurück, die das Ziel verfolgen, alle Brielwahlstimmen in den betreffenden Bezirken für ungülrig zu crklären. Zwar wurden Verstöße gogen das Wahlgesetz festgestellt. Allerdings ordneten die Richcer diese dem Grundsarz des cindeutig geäußerten Wählerwillens unter, denn trotz der Unterstützung durch republikanische Helfer gab es an der Auchentizirät der Stimmen keinen Zweifcl. ${ }^{16}$ Noch am selben Tag hob der Oberste Gerichrshof von Florida Richter Sauls' Urceil im wesenclichen auf und ordnete cine Nachzählung aller wundervores « in gesamten Staate Florida an. Die Frage, welche Markuerungen auf den Lochzerteln als gültigc Stimme zu werten seien, ließ das Geriche jedoch grundsätzlich offen. Stattdessen verwies es auf den im Recht des Staates Florida fest verankerten Grundsatz, daß jeder Stimmzettel zähle, der den Wählerwillen klar erkennen lasse. $\mathrm{D} a ß$ das Gericht nicht kontereter wurde, hängt gewiß auch damit zusammen, daß es andernfalls wiederum der unzulässigen richterlichen Rechtsfortbildung geziehen worden wäre. Die Anordnung des Nachzählens aller undervores schien dem Argument der Republikaner Rechnung zu tragen, daß eine Nachzählung nur in demokratischen Hochburgen unfair sei." Die Richter

1) Richeer Sauls, der von einem demokratischen Gouverneur ernanne wurde, galt nls der svahl konservativste Richeer in Leon County.

I4 Interessanterweise hatte Governeur George IW. Bush vor kurzer Zeit durch seine Unterschifí die Handauszählung zum bevorzugien Miucl der Stimmennachzählung in Texas Gesetz werden lassen, weil menschliche Augenscheinnahme zuverlässiger sei als das Sich-Verlassen aur Maschinen.

is Gore selbst unterstütne diese Klagen offiziell nicht, um nicht seine eigene Philosophie, daß zille Stimmen gezählt werden müßten, zu unterlaufen,

16 Diese Urteile wurden bald danach vom Florida Supreme Cour bestatigt.

17 Daß es sich bei der Beschwerde darüber, daß die Nachzählung nur in demokratischen Hochburgen unfair sei, in Wirklichkeit lediglich um cine Propagandaparole handelte und das Bush-Team cine Handauszählung in ganz Florida niemals ernsthaft wolle, wurde bereits wăhrend der ersten Verhandlung vor dem 
konnten sich bei dieser Anordnung auf eine breite Befugnis im Wahlgeserz snützen, wonach das Gericht alle geeigneren Maßnahmen anordnen kann, um Fehler bei der Wahl zu korrigieren. Außerdem wertete das Geriche die bei der ersten Handnachzählung bereirs als Scimmen für Gore identifizierten Stimmzettel als gültige Stimmen, so daß der Vorsprung Bushs bereirs vor der nun angeordneten manuellen Nachzählung auf weniger als zweihundert Stimmen geschrumpft war. Anders als die erste Enscheidung des Florida Supreme Court, erging dieses Urteil allerdings nur nit einer 4:3-Mehrheir. Während zwei Richter Rechtsfehler in der Enrscheidung des Tatsachengerichts erkannten, diese aber nicht für exheblich ansahen, sollte gerade der Präsident des Gerichrs, Chief Justice Wells, mit seinem harschen Minderheirenvorum Bush und dem U.S. Supreme Courr die Argumente für eine Aufhebung diescs Urteils mundgereche aufbereiten. Er schien die Aufhebung des Ureeils seiner Kollegen durch das Oberste Bundesgericht geradezu herbeischreiben zu wollen ${ }^{18}$ und äußerce die Befürchrung, daß die Verlängerung des gerichclichen Verfahrens das Land und den Scaat Florida in eine Verfassungskrise stürzen werde.

Bush griff zur leczten ihm zustehenden juristisclen Waffe und wandre sich - diametral enrgegengeseczt zu der von ihm sters verkündeten Philosophie - ar den United Stares Supreme Court als letzren Rettungsanker gegen die Macht des lokalen Gerichts. Er beantragte zunächst eine vorläufige Einstellung der Handnachzählungen, die mitclerweile zügig anliefen. Eine 5:4-Mehrheir des Gerichts erließ die gewünschte Anordnung und leitere damir den ersten Schritr zur Selbstkompromitrierung des Obersten Gerichtshof der Vereinigten Staacen ein. Eine solche Anordnung setzre nichr nur die Wahrscheinlichkeit eines Erfolges in der Hauptsache voraus, was an sich schon juriscisch reche fraglich schien. Sie erforderte auch, daß der Antragsteller deutlich machen konnte, daß ihm ohne Erlaß der einstweiligen Anordnung ein irreparabler Schaden drohe. Dies schien niche der Fall zu sein, denn der Supreme Courc konnte die bis zur Hauprsacheentscheidung vorgenommenen Nachzählungen für unrechrmäßig erklären und so den vermeintlichen Nachteil für Bush abwehren. Ein irreparabler Nachteil drohte vielmehr Gore bei Einstellung der Nachzählungen, denn nicht nur war Bush berejirs amtlich als Sieger festgestellt worden, auch bätce, wenn die Nachzählungen niche mehr rechtzeinig bis seclis Tage vor dem Zusammenrreten des Electoral College beender sein würden, ein positives Ergebnis für ithn nicht mehr der »sale harbor $\alpha$-Rcgelung seilhafrig werden können. Zudem hatte die republikanisch dominierte Legislative in Florida bereits Sondersitzungen eingeleitet, um angesichts der unsicheren Verhälınisse ihrerseits (auf Bush verpflichrce) Elcktoren für den Staat Florida zu bestimmen. Das Argument der republikanischen Mehrheirsführer war dabei, daß, wenn die Elektoren niche rechtzeitig sechs Tage vor dem Zusammentritr des Electoral College bestimmt seien, den sechs Millionen Wählern Floridas (von denen nur $48 \%$ für Bush gestimme hatten) die Aberkennung ihrer Stimmen, das sdisenfranchisemenc*, drohe, weil die Bestimmung der Elektoren danach für den US-Kongreß niclit bindend sei. Die Tatsache, daß hier unter dem Vorwand, das Wahlreche der Wähler Floridas zu sichern, Bush eine Versicherungspolice für den nicht unwahrscheinlichen Fall gegeben werden sollee, daß die Nachzählungen für ihn widrig ausgehen würden, ließ dic parteipolitische Zielrichnung nur

Florida Supreme Coun deutich, als dic Proxeßvereneung Bushs auf die Andcutungen des Goriches, cinc stnatswucice Handnachzählung zu erwägen, nicht cinging.

Is Wiclls: o sould not more strongly disagree with their decision... I also believe that the majority's decision cannot withstand the scruciny which will certainly immediately follow under the United Srates Constitution, $-\infty[N]$ o foundation in the law of Florida as in existed on November 7,2000 , or at any time unvil the issuance of this opinion. - [F] raught with equal protection concerns which will evcntually causc the election resules in Florida to be stricken by the fedenl courts or Congress. * 
allzu deuclich werden und kam auch in der amerikanischen Öffentlichkeit nicht sehr gur an. ${ }^{19}$ Allerdings retrete der U.S. Supreme Court den Geserzgeber Floridas schließlich vor diesem in Orwell'scher Manier bezeichneten Schrit, der den Wählern Floridas ihr Stimmrecht unter dem Vorwand entzogen härte, auf diese Weise Floridas Bevölkenung ihre Scimme bei der Präsidentenwahi zu crlaalıen.

Die mit 5:4 crgangene einstweilige Anordnung des U.S. Supreme Court zugunsten Bushs enthielt bereits ein von Richter Stevens auch namens Richterin Ginsburg und der Richter Souter und Breyer geschricbenes Minderheitenvotum. ${ }^{20}$ Interessant ist aber, daß sich Richter Scalia zu dem ungewöhnlichen Schritt bewogen fühlte, die Mehrheitsentscheidung mit einer eigenen Begründung (wconcurring opinion «) zu versehen. Seiner Meinung nach und - so darf vermuret werden - auch nach der nicht offen geäußerten Meinung der anderen Mehrheitsrichter würde das Zählen von zweifelhaften Srimmen irreparablen Schaden für George Bush und das Land bedeuten, da es einen Schatten auf die Legitimilät seiner Wahl werfen wirde. Die Stimmen erst auszuzählen und dann über deren Güluigkeir zu befinden, sei kein Rezepr, so Scalia, Wahlergebnisse zu produzieren, dic die öffencliche Akzepranz hätsen, welche die demokracische Stabilitäs crfordere. "Mit anderen Worten: Die vorläufige Anordnung war auch eine Vorsorge gegen die drohende Nichrakzeptanz des zu fällenden Urteils.

Was die Tatsache des Erlaßes einer vorläufigen Anordnung bereits andeutete, wurde durch die Hauptsacheentscheidung vom 12. Dezember bestäligt. Die konservative Mehrheit des Gerichts hob die Entsclzeidung des Obersten Gerichtshofes von Florida auf. Obwohl insgesame sieben Richter - einschließlich Soucer und Breyer-Probleme mit dem nicht einheitlichen Standard bei der Bewertung nicht vollständig ausgestanzter Lochkarten in den 2 s Distrikten mit Stanzkarten hatren, hielten nur fünf diesen Fehler für nicht behebbar. In ihrer nper curiam* ergangenen Mehrheitsentscheidung, die zwar den Verfasser niche sichtbar werden läßt, die aber die Handschrifo der beiden konservativen "swing votes $\alpha$, Sandra Day O'Connor und Anthony Kennedy, crägr, ${ }^{22}$ begründete die Mehrheit ihre Entscheidung mit einer Verletzung des bundesverfassungsrechtlichen Glcichheitssatzes (14. Verfassungszusatz), die darin liege, daß der Standard für die Handnachzählung (klar erkennbarer Wählerwillen auf dem Stimmzetcel) zu vage sei, wodurch Wählern in verschicdenen Bezirken eine unterschiedliche Behandlung ihrer Siimmzeteel zuteil würde. Dies bezog sich auf die Möglichixeit, ausgestanzte oder auch nur eingedrückte "chads" als gülrige Srimmen zu

is Wic heuchlerisch das Argument vom discnfranchisement der Wähler Floridas war, ist auch daran ersichtlich, daß die Infragestellung der nicht während der ssafe harbor *-Periode bestimnten Elektoren vom republikanisch dominieren US-Kongreß hïte kommen müssen, der Bush-Elekıoren wohl kaum beanstandet hatec, und daß dic Entrechtung der Wähler Floridas weit cklatanter gewesen wầre, wenn dic Legislative Bush-Elcktoren crnannt hätte, obwohl dic Auszählung der Stimmen eine Mehrhes fùr Gore ergeben hättc.

20 Richerin Ginsburg und Richter Breycr sind von Clinton emanne Richer, dic cinen gemäßigten Kurs richterlicher Zurückhalrung verfolgen und keinessegs als aktivistische liberale Richter gelten. Richter Soueer wurde von George Bush Sr. ernanne, ha sich aber als ein gemäßigter konservativer Liberaler enviesen, der angesichts der Rechıslastigkeir der fün (Mehrheitsrichter (Chief Justice Rehnquist, Richterin O'Connor, Richter Scalia, Kennedy und Thomas) als sehr liberal erscheint. Richter Stevens, von Ford crnannt, hat sich cbenfalls trozz konservativer Grundströmung als relativ liberal und unabhängig erwiesen.

21 Scalia wörlich. .The counting of votes that are of questionable legality does in my view tureaten irreparable harm to petitioner, and to the country, by casting a cloud upon what he [Bush] claims to be the legitimacy of his election. Count first, and rule upon legality aftervards, is not a recipe for producing election results that have the public aceeptance democratic stability requires. Diese Formulierungen Scalizs waren sicherlich auch eine Reaktion auf die Bemerkung in Richer Secvens' Minderhertenvotum, daß dic Verhinderung der Stimmenausxählung notwendigertveise einen Schatten auf dic Legitiritäı der Wahl werf́en werde.

22 Das ergibt sich auch daraus, dalis die anderen drei der Entscheidung ein separates Votum beifügten, in dcm sie noch weitergehende Gründe für dic Aufhebung der Entscheidung des Florida Supreme Court anfüliren. Siehe dazu unten Fn. 24. 
werten, je nach Urteil des betreffenden Wahlausschusses. Außerdem wurde ein anderes Argument aus dem Minderheitenvotum von Chief Justice Wells zu dem beanstandecen Urteil des Florida Supreme Court aufgegriffen, nämlich die Tatsache, daß lediglich »undervores" nachgezählt werden sollten, nicht aber die insgesamr ca. i 1000 * overvores", also Srimmzertel, die als ungültig gewerret wurden, weil mehr als ein Präsidentschaftskandidar markiert war. Dies ist indes cin äußerst formaliscischer Einwand, denn das Problem in den Stanzkartenbezirken, die im Fokus der Betrachtung lagen, war, daß die Maschinen nur deutlich ausgestanzte "chads" als gülcig registrierten. Wo die Maschinen aber bereits mehr als eine Stimme zählten, war die Wahrscheinlichkeit, daß sich bei Augenscheinnahme des Scimmzertels ein klarer Wählerwille für nur einen Kandidaten finden ließe, denkbar gering. "3

In der Tar stimmtc es bedenklicl, daß verschiedene Wahlbezirke oder gar Wahlauszähler unterschiedliche Maßstäbe bei der Frage anlegten, ob ein klarer Wählerwille erkennbar sei. Allerdings konzentrierte die Mehrheit des U.S. Supreme Courr ilare Gleichheitsprüfung auf eine Mikroanalyse. Die Makroanalyse hätre gezeigts daß die Handnachzählung der undervores eigentlich nur ein Mittel war, eine weit größere Gleichheirsverlerzung zu kompensieren, die dadurch encstand, daß unterschiedliche Stimmzettel und Auszähiverfahren in Floridas Wahlbezirken verwandt wurden, die zur Folge hatten, daß für Wähler in den 40 Bexirken, in denen optische Lesegeräre vcrwandt wurden, eine weit höhere Chance bestand, daß ihre Stimmen korrekr gewertet würden. Die abgeschlossene Handnaclizähiung in Broward County ergab, daß dort auch nachher die Anzahl der "undervores" immer noch um ein Vielfaches höher blieb ais in Bezirken mit oprischen Lesegeräten. Diescn Umstand ignorierend, beraubten die Mehrheitsrichter stattdessen diejenigen Wähler, deren Wille vielleicht bei manueller Nachzählung noch cindeutig zu ermitzeln gewesen wäre, einer solchen Chance mit dem Hinweis darauf, daß diese Chance wegen des uneinheitichen Standards bei ihnen untereinander ungleich groß sci. Die Mehrheit des Suprenie Courc beschränktc ilurc Betrachtung aber nicht nur auf den Mikro-Gleichheitsverstoß eines ungenauen Standards, sondern folgerte gleicl auch noch - olne eine Tatsachengrundlage darüber in den Akren zu haben-, daß dieser Verstoß niche durch eine neuc Anordnung des Obersten Gerichis von Florida in der Kürze der Zeit zu korrigieren sei. $^{24}$

Richter Srevens wics in seincm Minderheitenvorum (Richter Souter, Richterin Ginsburg und Richter Breyer verfaßten weitere Minderleitenvoten) darauf hin, daß eine Mehrlseit der Einzelstaaten denselben Standard bei der Ermittlung des Wählerwillens zugrunde lege, der nun von der Mehrheit seiner Kollegen als Gleichheitsverstoß angesehen werde. Er machce auch den naheliegenden Verweis, daß dem Kalkül der Mehrheit zufolge auch der bei strafrechrlichen Verurteilungen zugrunde gelegte Standard ("no rcasonable doubc ") einer Gleichheitsprüfung kaum standhalte. Srevens sah die Bush-Klage gänzlich auf dessen Mißtrauen gegenüber den Richtern des Staates Florida fußend, die die Auszählung der Stimmen überwachen würden. $D_{a} ß$ die Mehrheit seiner Kollegen diesc Sicht mir ihrer Entscheidung stütze, werde zu

23 Fin weiteres Scheinargument diescr Ars war, daß einige Wähler womöglich zwei Scimmen abgegeben haben könnten, von deren aber nue eine von der Maschine regiserierı rvorden scin könnte, so dak ihre Stimınc zu Unrechı als güleig angesehen roorder sein kónnce.

24 Die drei konservativsten Richter am Suprense Court anikulieren in einem van Chief Justice Rehnquist gezcichnceen Separatvotum weitere Gn̈nde, rvarum das Ureil aus Florida gegen die Bundeswerfassung verstofe. Das Entscheidende für sic war, daß die Richer cin gescexgeberisches Schema aus den Angaln gehoben und somit unzulässige richterliche Rechtsfortbildung geübt häuen und zu einer Auslegung gelang seien, der a keine vernünftige Person a folgen könne. Gleıchzeisig erhoben sie die vom US-Konereß (und nicht von der US-Verfassung) kreiene ssafe harbor -Frist \%u viner quasi-konsticutioncillen Vorgabe, die zwingende Wirkungen auf das Wahlprüfungsverfahren in Florida cntfalte. 
einer zynischen Einstellung gegenüber den Richtern im ganzen Land führen. ${ }^{\text {is }} \mathrm{Er}$ schloß sein Votum mit dem Satz: "Obwrohl wir womöglich niemals mir absoluter Sicherheit wissen werden, wer der Gewinner der diesjährigen Präsidentschaftswahl war, die Identität des Verliercrs ist nur allzu klar. Es ist das Vertrauen der Nation in einen unparteiischen Hüter des Rechtssraats. "

Insgesamt war die juristische Grundlage, auf der der Supreme Court eine äußerst knappe Entscheidung weitreichenden politischen Ausmaßes traf, äußerst dünn. Niemals zuvor hatre das Gericht mir seiner Entscheidung eine Wahl entschieden. Niemals hattc es die Wahlmodalitäten in einem Einzelstaat einer Nachprüfung unterzogen. Die nunmehr gemachte Ausnahme wurde unter Bcrufung auf nicht cinschlägige Präzedenzien unter Zugrundelegung ciner für den konkrcren Fall geschaffenen Gleichleitssatzdogmatik gemacht, was den Abgrund, in den sich der Supreme Cour begab, nur noch deutlicher werden läßt. Die solche Vorwürfe nicht leichtfertig äußernde liberale New Republic sah den Supreme Court als ein auf Seiten der Republikaner stehendes parteiliches Organ und folgerte: $n$ Verfassungsrechclich gesehen ist diesc Präsidentschatt auf unrechtmäßige Weise erlangt [ill-gotten]. Sie ist der Preis eines justiziellen Putsches. : $^{\circ}$ Angesichrs dessen verwundert es zu lesen, daß Josef Joffe die Entscheidung des Supreme Court in der ZEIT quasi als einen konscnssriftcnden Akt interpretiert."

Gore hatte im Vorhinein geäußerr, daß cr eine Entscheidung des Supreme Court akzeptieren werde. Während die Republikaner die ihnen widrigen Entscheidungen des Florida Supreme Court als Dezisionen parteilicher Richter brandmarkten und niemals den Eindruck erweckten, als wenn sie sich mit einem Ausgang zugunsten Gores abfinden würden, ${ }^{28}$ spielte Gore nach leichtem Druck aus seiner Partei den ritterlichen Verlierer und kanzidierte seine Niederlage. Die amerikanische Ölfentlichkeit erweckt den Eindruck, als ob sie sich mit dem Bush-Sieg arrangieren kann. wie sie wohl auch einen Gore-Erfolg angenommen hätte. Und schon scheint sich alles wieder in Richtung Normalitär zurück:zuentwickeln. Aber wern es um demokratische Legitimitär geht, bei dem muß dic Art und Weise, wie um das Präsidentenamt gestritcen und wie die Entscheidung letztlich getroffen wurde, schwere Bedenken daran aufkommen lassen, wie Demokratic in Amerika praktizien wird. Die Ereignisse zeigten zıvar auch eine bedauernswerze Qualitär des rechnischen Geräts an, das den Wahlausschüssen zur Durchführung der Wahlen zur Verfügung stand und das offensichtlich nicht geeignet war, ein auch nur einigermaßen verläßliches Ergebnis zu

25 Ricluer Stevens zeigte auch auf, daß der in Art. II $\$$ - US-Verfassung bezcichnete Gesetzgeber nicht als losgelös vom Recht des betreffenden Gliedstaates begriffen werden könne und daß die $\gg$ sale harbor Regel keinen Verfassungsrang genieke. Richter Sourer, der sich in Gegensatz zu den anderen beiden Minderheitsrichern Stevens ' Votum nicht ausdrücklich anschloB, obwohl er ihm in wesentlichen zustimmte, sahes als verfehlt an, daß das Gericht den Fall überhatspt xur Entscheidung angenonimen habe. Wie auch Richter Breyer in seinem separaten Minderheitenvotum sab er den möglichen Glejchheitsverstoß als noch heilbar an. Richterin Ginsburg beklagec unter anderem, daß die von der Mehrhcit angeführten Präzedenz-Fälle die Tatsache verschleicnen, daß dic Entscheidung sich in Wirklichkeit nicht. auf die bisherige Kasuistik stützen lasse. Ginsburg wies auely auf die Skurrilität hín, daß sich unter den spärlich angefuhren Fällen, mit denen nun begnündes wurde, daß eine Reihe von Seimmen in Flosida niche mehr gezählt werden sollten, Fälle waren, mit denen der Supreme Court scinerzcit gegen den Ausschiuß von Schwarzen vom Wahlreche entschicden hate.

26 Unsafe Harbor, The New Republic, Dec. 25, 2000, S. 9.

27 Amerikas Exblolgekrieg, Die Zeil Nr.s1 s. 14.12.2000, S. 1. Joffe verkennt auch, daß es eine Reihe von Entscheidungen des Supreme Court gab, dic von der Bevölkerung nicht akzcpticrt wwurden und keine Befriedung, sondern cine weitere Spaltung bewirkten. Genannt scien erwa Dredd Scolt v. Sarford, 60 U.S. 393. $19(18,6)$, wo festgestelle wurde, daß die Schwarzen keine Stazesbuinger scien; Plessy v. Terguson, 163 U.S. $537,16(1896)$, wo die Rassentrennung aufrech erhalren wurde, und Locloner v. New York, i98 U.S. 43, 2s (1905), und im Gefolge weitere Entscheidungen des damaligen konservativen Cicrichus bis zum New Deal, miz denen die Sozialgeserzgcbung in den USA blockiert wurde.

28 So wurde bereits die ldee kolporsiers, in eınem solchen Fall dis Inaugurationsfeierlichkeiten zu baykotricren. 
liefern. ${ }^{2 y}$ An diesem Manko werden die polirisch Verantworclichen sicherlich arbeicen. Weic fundamentaler ist die sich aufdrängende Frage, ob eine Wahl in einem sich demokratisch nennenden Staat durch die Wähler legitimiertes Anvercrauen von Macht bedeuten soll oder nur eine letztlich belicbige Methode darstell, die Regierungsverantwortung auf irgend jemanden zu übertragen. Aus Sicht der Republikaner jedenfalls tat das zurage tretende Legirimationsdefizit ihrem Machtanspruch keinerlei Abbruch. Dabei gescanden selbst beinharce Republikaner ein, daß ilar Präsidentschafrsbewerber kein Manda der amerikanischen Wähler für die Umsetzung seiner programmatischen Vorstellungen bei dieser Wahl erhalten hatte, die eher einem Unentschieden gleichtam. ${ }^{30}$ Zwar hat Bush versprochen, eine Politik über die Parreigrenzen hinweg betreiben zu wollen. Dessen ungeachter macht er sich aber bereits daran, seine im Wahlkampf versprochenen umstrittenen Sceuerpläne umzusetzen. In sein Kabinerr hatte Bush zunächst zwar hauptsächlich gemäßigte Konservative berufen. "Mit Linda Chavez für das Arbeirsminisrerium und John Ashcrofr als Attorney-General nominierte er dann aber auch zwei Konservative, die für Posirionen stehen, die mit Sicherheit nicht konsensfähig sind. ${ }^{3 x}$ Man braucht kein Prophet zu sein, um vorauszusagen, daß die Ideen der $52 \%$ Wähler, die nicht für ihn scimmten, in seiner Administration wenig Berücksichrigung finden werden. Trotz des fehlenden Mandats wird der Präsident so handeln, als ob er zur Durchsetzung eines - wenn auch vielleichı gemäßigteren - konservativen Programms legicimiert wärc.33 Der im amerikanischen System sowieso diffuse Wählerwille, der - soviel läßr sich sagen - arm ehesten eine zencristische Linie gebicten würde, wird kaum noch gebraucht werden, um Regierungsentscheidungen zu legitimieren.

Daß etwas falsch am amerikanischen Regierungssystem sein könnte, wird man sich bald kaum noch fragen. Dabei ist es nur zu deurlich geworden, daß es keine Institurion gibt, der die Bürger Unparteilichkeit zutrauen. Die amerikanische Merhode, Wahlen als Legitimazion dafür aufzufassen, dic gesamte Regierungsverantwortung an parteiliche Funktionäre des Siegers zu übergeben, hat eigentlich seine daraus resul-

29. Wie in anderen offentlichen Angelegenheiten, die in der finamziellen Verantwortung der Gemeinden und Bezirke liegen (siche erwa das Schulwesen), waren es auch hier vorwiegend weniger reiche Gchictskörperschaften, die nicht auf dem optimalen technischen Stand waren.

10 Auch das Ergebnis der Kongreßwahlen sicht eher wie ein Unentsehieden aus. Die republikanische Mehrheit im Repräsentantenhaus ist auf wenige Stimmen zusammengeschrumpft; der US-Senat wcist cin 50:50-Patt zovischen Republikanern und Demokraten auf, wobei jedoch zu beachten ist, daß der Stichentscheid bei Stimmenglcichheit dem Vizepräsidenten, also Dick Cheney, zukommt. womit sicls de facto ein Übergewicht der Republikaner ergibe.

31 Auch cin Demokrat erhich cinen Kabinettsposten. Der erste Schritt Bushs, einen parciubergresfenden Eindruck zu machen, bestand jedoch in dem Versuch, cinen demokratischen Senator aus Louisiana für scin Kabinett zu gewinnen. Dies hätte das gegenwärtige Patt im Senat in cine Mehrbeit der Republikaner verwandelt, da der Nachfolger durch einen republikanischen Gouverneur bestimnı worden wäre.

32 Linda Chavez, eine notorische Gewerkschafusfeindin, dic überdies viele der Gesctze, auf deren Einhaltung das Arbeitsministerium zu achen hat, prinzipiell ablehne, mußte ilare Kandidatur inzwisehen wegen der nicht offengelegten früheren Beschäftigung einer illegalen Ausländerin in ihren. Haus zurückrjehen. John Ashcrof, der neue Atorney-General, ist ein dezidierter Aburcibungsgegner, der șelbst die kriminologische Indikation (z. B. bei Schwangerschaft nach Vergewaltigung) ablehnt, und ise darüber hinaus ein ausgesprochener Schußwaffenadvokat. Als amerikanisches Kuriosum sollte nicht uncrwähn bleiben, daß Ashcroft zuvor bei dem Versuch, wieder in den US-Senat gewählt zu werden, cinem Toten unerlegen war. Sein Gegenkandidat, Mel Carnahan, kam kurz vor der Wahl bei cinem Flugzeugunfall um, erhieht aber die Mehrheit der Stimmen bei der Wahl, nachdem der demokratische Gouverneur von Missouri, der dafür zustandig ish, einen Nachfolger für den vakanten Senatsposten scines Staats zu ernennen, angekündigt hatec, Carnahans Witwe zu benennen, wenn deren verstorbener Mann mehr Stimmen als Asheroft crhale und der Senusssitz sumit vakant blcibe.

13 Bezeichnend für dic republikanische Hypokrisic war es, daß Gore vorgeworfen wurde, er unterminiere unit seinen Aktionen die Legitimität der Präsidentschaft und schade so dem Staat, rachdem die republikanische Kongreßłführung Prisident Clintons Präsidentschaft während dessen gesamter Amtszcil mit allen Mitteln zu deligitimieren versucht hatte und sich daber auch niche gescheut hate, cin von vornherein aussichus- und basisloses Impeachment-Verfahren gegen ihn einzuleiten. 
cierenden Schwächen zur Genüge offenbart. Warum, so muß man als halbwegs Außenstehender fragen, müssen Wahlvorsünde fast ausschließlich mit Angehörigen der Mehrheitspartei besetzt werden, so daß die andere Partei diesen den guten Willen von vornherein absprechen kann? Warum müssen Richter sters vom jeweils regierenden Gouverneur oder Präsidenten ernannt werden, so daß diesc Ernennung ihnen später als Zeichen ihrer fehlenden Neucralität vorgehaiten werden kann? Warum ist eine Union, die sich im Laufe von über zweihunder Jahren längst zu einer Nation entwickele hac, nicht in der Lage, für ihren Präsidenten, der als einziger in einem einigermaßen nacionalen Wahlkampf ermictelt wird und daher der cinzige wirkliche Repräsentant des gesamten amerikanischen Volkes isı, ein einheitliches Wahlverfahren zu schaffen, das elementaren demokratischen Gepflogenheiten Rechnung erägt? Vicles hängı mit der Immobilitär des hochgepriesenen Verfassungswerks zusammen, das Änderungen quasi nicht zuläßt. ${ }^{j+}$ Insbesondere die leczte Frage ist aber nícht nur für Amerikancr von Interesse, sondern z. B. auch für Europäer, die an der Fortentwicklung ihrer Union arbeiten.

Daß viele Amerikaner stolz davon sprechen, daß es keine Verfassungskrise gegeben habe, da anders als in anderen Ländern keine Panzer in den Straßen gestanden hätren und der Konflikt durch die Gerichte gelöst worden sei, klingt geradezu lächerlich. in Wirkliclukeit hat die »rule of law*, das Rechissraasprinzip, durch die Art und Weise, wie der U.S. Supreme Court seine policische Entscheidung nur spärlich mit dem Mäntelchen des Rechrs verhüllt hat, dauerhaften Schaden genommen. Künftig werden es nicht nur politische Amtsträger und ortliche Richter sein, die man nur noch als Parteisoldacen wahrnimms, sondern auch die Richter des U.S. Supreme Court," die bisher - wohl zu Unrecht - noch am ehesren Versauen in ihre Unparceilichkeir genossen.

34 Verfassungsänderungen erfordern nicht nur einc 2/3-Mchrheit in beiden Häusern des Kongreßes, sondern auch cinc Ratifizierung durch 3/4 der Einzelstaaten (siche Art. V US-Verfassung). Dies crzeugt de facto cin kaum zu überwindendes. Hemmnis für Änderungen, die dic Macha der kleineren Staaten begrenzen wïrden.

3s Nicht unerwähnt bleiben sollte, daß Bush während seiner vierjährigen Amtszeit wonöglich bis zu drei Richterstellen am Supreme Court wird neu besetzen könmen. Es wird allgemein angenommen, daß Chief Justice Rchnquist, der, noch von Nixon ernannt, dem Gericht seit 1972 angehört, und Richterin $\mathrm{O}^{\prime} \mathrm{Connor}$ in den nächsten Jahren freiwillig ausscheiden werden, um einem republikanischen Präsidenten die Möglichkeit zu geben, ihre Nachfolger zu bestimmen. Auch der 8o-jährige Richter Stevens ist seit 1972 Mitglicd des Gerichts. Sollec seine Stelle während Bushs Amtszeit neu zu besetzen sein, würde dies die bisherige konșcrvative Mehrheit womöglich von $5: 4$ xuf $6: 3$ vergrößsem. 